\title{
INVESTIGACIÓN DE AUDIENCIAS EN LAS SOCIEDADES DIGITALES: SU MEDICIÓN DESDE LA PUBLICIDAD
}

\section{Audience research in digital societies: Their measurement from advertising point of view}

Natalia Papí-Gálvez y Marta Perlado-Lamo-de-Espinosa
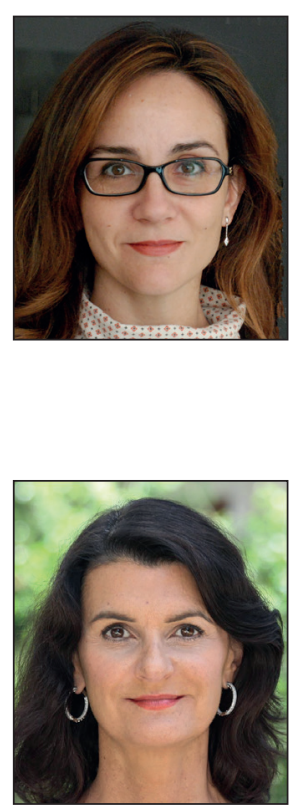

Natalia Papí-Gálvez es titular de universidad en la Universidad de Alicante. Profesora de Investigación y Planificación de Medios Publicitarios desde 2001, es directora del Grupo de estudios sobre comunicación estratégica (E-COM). Una de sus líneas de investigación es la investigación, estrategia y planificación de medios publicitarios, con especial atención en los nuevos medios y la comunicación digital. Actualmente desempeña el cargo de Vicedecana del Grado en Publicidad y Relaciones Públicas.

http://orcid.org/0000-0002-4871-1691

Universidad de Alicante Facultad de Ciencias Económicas y Empresariales, Departamento de Comunicación y Psicología Social Campus de Sant Vicent del Raspeig. 03690 Alicante, España natalia.p@ua.es

Marta Perlado-Lamo-de-Espinosa es profesora contratada doctora en la Universidad Antonio de Nebrija. Profesora de Investigación de Audiencias y de Planificación de Medios desde 1995, actualmente es decana de la Facultad de Comunicación y Artes de la misma universidad. Miembro del grupo de investigación en comunicación (Inecom) de la Universidad Antonio de Nebrija, sus últimas publicaciones están enfocadas al estudio de audiencias y medios de comunicación y al análisis de las competencias y perfiles profesionales en la comunicación.

http://orcid.org/0000-0002-7476-0809

Universidad Antonio de Nebrija Facultad de Comunicación y Artes, Departamento de Publicidad C/ Sta. Cruz de Marcenado 27. 28015 Madrid, España mperlado@nebrija.es

\section{Resumen}

La investigación de audiencias es útil para la publicidad. Facilita la toma de decisiones sobre los medios publicitarios más idóneos para hacer llegar una campaña, aunque la utilidad de los datos que proporciona también depende del modelo comunicativo imperante. De hecho, el componente digital y online del ecosistema mediático actual precipita un nuevo paradigma, pues cuestiona el modelo tradicional de medición de las audiencias. Este artículo explora y describe la información recogida por los estudios de audiencias de 23 países con especial atención en España. Los resultados son discutidos a la luz de las demandas de medición de las audiencias del mundo digital.

\section{Palabras clave}

Publicidad; Medios; Investigación de medios; Investigación de audiencias; Audiencias online; Comunicación publicitaria; Planificación de medios; Fuentes internacionales; Indicadores; Indicadores sociales.

\begin{abstract}
Audience research is useful for advertising. It facilitates the decision making on the best advertising media to spread a campaign, although the usefulness of the data it provides also depends on the dominant communicative model. In fact, the digital and online component of the current media ecosystem precipitates a new paradigm, as it questions the traditional model of audience measurement. This article explores and describes the gathered information by the audiences studies of 23 countries, with special attention to Spain. The results are discussed in light of the demands of audience measurement of the digital world.
\end{abstract}

\section{Keywords}

Advertising; Media; Media research; Audience research; Online audiences; Advertising communication; Media planning; International sources; Indicators; Social indicators. 
Papí-Gálvez, Natalia; Perlado-Lamo-de-Espinosa, Marta (2018). "Investigación de audiencias en las sociedades digitales: su medición desde la publicidad". El profesional de la información, v. 27, n. 2, pp. 383-393.

https://doi.org/10.3145/epi.2018.mar.17

\section{Introducción}

La investigación de audiencias analiza la exposición de la población a los medios de comunicación, por lo que contribuye a un mayor conocimiento de los mismos. De estos estudios se desprenden mediciones, estadísticos e indicadores que pretenden cubrir la demanda de información de las empresas periodísticas y publicitarias. Su utilidad también depende de las necesidades de medición de las sociedades actuales.

En publicidad los estudios de audiencias son claves para poder dirigir la comunicación al grupo de interés para el anunciante. Conseguir que la publicidad tenga la oportunidad de contactar con el público objetivo es responsabilidad de la planificación de medios publicitarios (Perlado-Lamo-de-Espinosa, 2006; González-Lobo; Carrero-López, 2008; Papí-Gálvez, 2017). Este ámbito específico de la publicidad, sobre todo cuando trabaja con los medios de comunicación preinternet, se caracteriza por la aplicación de técnicas que utilizan el dato de audiencia para seleccionar los soportes en los que insertar el anuncio. El mundo digital conectado a internet contribuye a ampliar el enfoque de la publicidad y como consecuencia, el de la planificación de medios (Solana, 2010; Papí-Gálvez, 2014).

La tecnología flexibiliza procesos y formas de trabajo offline. La digitalización de los medios clásicos o tradicionales abre otras posibilidades. Por ejemplo, ofrecer contenidos directamente al usuario (como sindicación o descargas de podcast o vodcast), que puedan ser difundidos y consumidos en tiempo real o en diferido (streaming video o viewed on same day as live- Vosdal) y espacios para conversar o compartir contenidos (blogs).

A estas posibilidades se suma la diferente oferta de soportes. Los medios de comunicación presentes en internet se multiplican y a los tradicionales, adaptados al medio online, se suman otros nativos (Campos-Freire, 2015). Aparecen los buscadores y los medios sociales con su particular modelo de negocio y la publicidad adquiere otras formas relacionadas con las posibilidades de cada soporte. Incluso cuando se trata de publicidad display, fórmula que se aproxima en mayor medida a la manera tradicional de entender la publicidad, esta adquiere mayor fluidez. En internet la publicidad puede rotar, aparecer y desaparecer en tiempo real entre la multitud de soportes que lo forman.

A lo anterior hay que añadir el aumento de la exposición a varias pantallas o dispositivos, que desemboca en otras formas de consumo combinado, ofreciendo nuevas categorías de audiencias, como las sociales (Papí-Gálvez, 2015a; Sánchez, 2017).

La inclinación por conocer el tamaño y perfil de la audiencia nace en el mismo momento en el que aparecen los medios de comunicación social (Huertas, 2002). La creciente competitividad entre las empresas mediáticas por la audiencia y por los ingresos derivados de la publicidad, es fuente de motivación para estos análisis (Wimmer; Dominik, 2001). Igualmente el interés de los publicitarios en las décadas de los cincuenta y sesenta por los resultados de estos estudios para su propio campo de actuación profesional es uno de los factores esenciales para el desarrollo de la investigación empírica de los medios de comunicación. No obstante, la expansión del mercado de los medios de finales de los ochenta representa el mayor aliciente para el progreso de la medición de audiencias, al menos en Europa (González-del-Valle, 2003). Este avance se sustenta en la importancia que los agentes del sector (medios, anunciantes y agencias) otorgan al dato de medición. La audiencia es considerada necesaria incluso para la propia práctica periodística (Masip, 2016).

Con la llegada de las nuevas tecnologías se abre el debate sobre la idoneidad de las herramientas habituales de medición de audiencias para poder observar los nuevos escenarios (Livingstone, 2004). Las peculiaridades del ecosistema digital online implican la demanda de mediciones ajustadas a la nueva realidad que faciliten a la publicidad la toma de decisiones. El modelo de medición de las sociedades digitales debería poder capturar datos en cualquier momento, lugar o dispositivo, y para cualquier contenido (Madinaveitia, 2013; Madinaveitia; Merchante, 2015).

La investigación de audiencias actual se basa en gran medida en la encuesta, técnica centrada en el usuario. Este método de investigación social es aplicado con rigor y amplio consenso por parte del sector (Lamas, 2004), pero sus limitaciones son también conocidas. Con el objeto de salvar algunas de ellas, el sector de la investigación de medios, representando por entidades como la española Asociación para la Investigación de Medios de Comunicación (AIMC), lleva estudiando durante años las bondades de la medición electrónica (Lamas, 2002). Sin embargo se ha aplicado solo a mercados cuyo volumen de negocio haga rentable su puesta en marcha, como es la televisión.

La medición electrónica está ganando terreno en internet (Lamas, 2010). En España se propone un sistema híbrido parecido al llevado a cabo en el audiovisual (Papí-Gálvez, 2015a). Este diseño podría perfeccionarse a la luz de los futuros avances tecnológicos, y a juzgar por el debate en torno a la gestión y el valor de los datos masivos (Hill, 2014; O’Hara, 2016; Nelson; Webster, 2016; Papí-Gálvez, 2015b). Igualmente el modelo basado en la fusión de paneles muestrales de distintos dispositivos, con bases censales distintas, ya empieza a funcionar en países como Noruega, Suecia, Alemania, Holanda o Suiza (Santiago, 2017).

El ámbito de la investigación de medios en general y el publicitario en particular, se encuentra en un contexto sin precedentes. Aparece un nuevo paradigma de medición/ investigación, en paralelo a la mutación del modelo comuni- 
cativo, evidenciando que la medición tradicional (muestras o estudios single source) no captura la complejidad del entorno digital actual, pues precisa la integración de fuentes distintas. La implantación y el desarrollo de los diferentes modelos de medición de audiencias son continuamente objeto de debate en foros nacionales e internacionales.

Con este propósito destaca a nivel internacional EMRO (European Media Research Organisation). Se registró como asociación en 2011, aunque sus orígenes se remontan a 1960. Actualmente está formada por entidades directamente relacionadas con la medición de audiencias que proceden de 23 países, entre ellos España. Desde 2015, con motivo del encuentro anual, se presenta y actualiza el inventario de fuentes de investigación de medios EASI (Emro Audience Survey Inventory), que reúne información descriptiva de tres años sobre los estudios de audiencias por medios y países y constituye una fuente única que facilita la comparación de la medición española respaldada por AIMC con la de otros países ${ }^{1}$. Esta fuente es clave para conocer el estado de la investigación de audiencias desde una perspectiva internacional y reflexionar sobre los desafíos de medición digital.

Durante años la investigación de audiencias ha sido revisada y ampliada, previo debate y consenso, también en lo que concierne al medio internet.

Ante este escenario se explora la información que proporciona la investigación de audiencias según la recogida en $E A S I$, atendiendo al marco de las sociedades digitales y desde una perspectiva publicitaria. En concreto este estudio pretende:

- detectar la cobertura de la investigación de audiencias en términos de tipos de estudios realizados por países;

- revelar y agrupar la información clave de cada estudio para dimensionar los indicadores;

- identificar los principales rasgos de la investigación de audiencias internacional;

- conocer la situación de la investigación de audiencias española en el contexto internacional.

\section{Método}

Se planteó un exploratorio de los estudios de audiencias y un descriptivo de la información contenida. Se analizó el último inventario EASI de EMRO correspondiente a 2017 con 23 países, que actúa como fuente de información secundaria para esta investigación ${ }^{2}$.
Esta fuente contiene un primer bloque que incluye 36 preguntas sobre la cobertura de la medición de los medios por países. Además de las 2 preguntas identificativas, queda formado por:

- 5 preguntas básicas sobre la medición de televisión (Television audience measurement o TAM);

- 3 preguntas sobre la de radio (Radio audience measurement o RAM);

- 7 sobre la encuesta de medio impreso (National readership survey o NRS);

- 2 sobre la medición del medio exterior;

- 5 relacionadas con cine;

- 6 cuestiones básicas sobre la medición del medio internet;

- 2 sobre la existencia de un estudio multimedia (cross media o CMM);

- 4 relacionadas con la determinación de públicos objetivos (target group o TG).

El inventario también está formado por otras 6 secciones que profundizan en aspectos concretos de cada estudio o medio, excepto del cine y TG. Cada sección presenta un número concreto de preguntas (tabla 1). Todas comparten el país y la fecha de cumplimentación del cuestionario como variables identificadoras o de control.

EASI facilita observar la consistencia del recurso al comparar las respuestas de algunas preguntas de la sección general con las de las partes siguientes. Se aprecia que todos los países responden a la primera sección (tabla 1 ) y que la gran mayoría de los no presentes en la segunda parte del cuestionario se debe a que no disponen de estudios. No obstante también existe una pérdida de información sin causa aparente.

- en televisión Dinamarca, Francia y Sudáfrica dejan de contestar la parte específica, pese a declarar disponer de TAM;

- en radio no responden Dinamarca y Rumanía;

- Noruega no cumplimenta el apartado del medio impreso;

- Francia y Suecia no están en la sección específica del medio exterior:

- dejan de responder el cuestionario sobre el digital online cinco países (Bulgaria, Dinamarca, Países Bajos, Sudáfrica y Suecia);

- la sección CMM también deja de ser contestada por Dinamarca y Francia; y se detecta una anomalía, Bélgica, pues en este caso ocurre lo contrario, es decir, pese a declarar no disponer de cross media cumplimenta la parte del CMM con los datos de un año anterior.

Tabla 1. Número de preguntas y países que responden por sección

\begin{tabular}{|l|c|c|l|}
\hline \multicolumn{1}{|c|}{ Sección } & $\begin{array}{c}\text { Preguntas/ } \\
\text { cierres }\end{array}$ & $\begin{array}{c}\text { Países que } \\
\text { responden (n) }\end{array}$ & \multicolumn{1}{|c|}{ Países que no responden } \\
\hline General & 36 & 23 & Ninguno \\
\hline Televisión & 78 & 20 & Dinamarca, Francia, Sudáfrica. \\
\hline Radio & 68 & 21 & Dinamarca, Rumanía. \\
\hline Medios impresos & 74 & 20 & Bulgaria, Marruecos, Suecia. \\
\hline Exterior & 63 & 11 & $\begin{array}{l}\text { Bulgaria, Eslovenia, Finlandia, Francia, Grecia, Marruecos, Noruega, Polonia, Portugal, } \\
\text { Rumanía, Rusia, Suecia. }\end{array}$ \\
\hline Cine & No hay & No hay & No hay \\
\hline Digital online & 74 & 17 & Bulgaria, Dinamarca, Marruecos, Países Bajos, Sudáfrica, Suecia. \\
\hline Cross media & 74 & 17 & Bulgaria, Dinamarca, Francia, Marruecos, Polonia, República Checa. \\
\hline
\end{tabular}


El recurso proporciona datos de varios años, según la última respuesta y actualización realizada por los países.

Para dar respuesta al primer objetivo se observó la información solicitada por la fuente y las respuestas de cada país, sobre todo de la sección general del inventario. Se realizó la cuantificación de los estudios y países y se extrajeron las frecuencias.

Para el segundo y tercer objetivo fueron revisadas las partes que profundizan en las características de los diferentes estudios por separado. Se aplicó una aproximación cualitativa y cuantitativa al análisis de cada parte. Para el segundo objetivo se relacionaron y agruparon las preguntas de las seis secciones según la información que extraían, incorporada en la primera de las columnas de cada sección, y los cierres, observados en el resto de columnas. Para el tercero, se seleccionaron las cuestiones que más respuestas concentraban y se calcularon las frecuencias.

Finalmente, para alcanzar el cuarto objetivo se situó a España en cada análisis de los datos realizados en todas las secciones del inventario.

\section{Resultados}

\subsection{Cobertura de medios por países}

Los 23 países tienen algún estudio para conocer las audiencias de televisión y radio (figura 1 ). Es frecuente que sean llevados a cabo por un único operador. No obstante la mayoría no introducen la retransmision en directo (streaming video) en el estudio de medición de la televisión. La mitad lo incluyen en la encuesta de internet (11 países).

Prácticamente todos los países recogen audiencias del medio impreso $(n=21)$ en el que se puede observar de forma conjunta la audiencia de los diarios y las revistas. La ex-

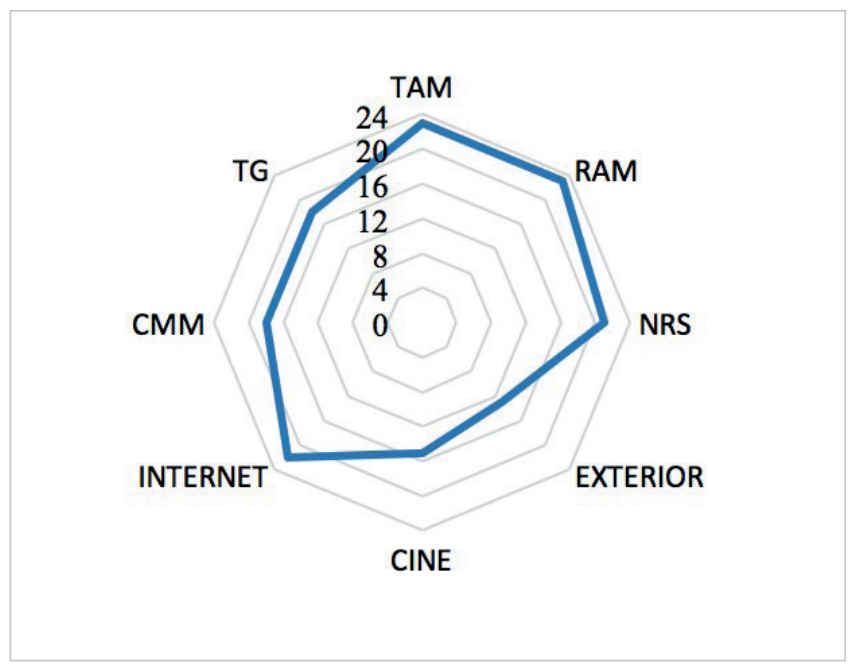

Figura 1. № de países por estudios de audiencias Fuente: elaborado con datos de EMRO (EASI, 2017)

cepción se detecta en Bulgaria y Marruecos, pues ninguno dispone de una encuesta que mida este medio. Marruecos tampoco cubre el medio internet. 17 países preguntan por el uso del móvil en las encuestas de internet.

El medio exterior es abordado por un estudio especializado en 13 países. No se observa en Bulgaria, Eslovenia, Finlandia, Grecia, Marruecos, Noruega, Polonia, Portugal, Rumanía y Rusia (figura 2).

Tan sólo Austria, Reino Unido y Suiza disponen de un estudio específico de cine, aunque el medio queda integrado en otras encuestas en 12 países más $(n=15)$. Bulgaria, Finlandia, Grecia, Marruecos, Polonia, Portugal, República Checa y Ucrania no hacen medición alguna.

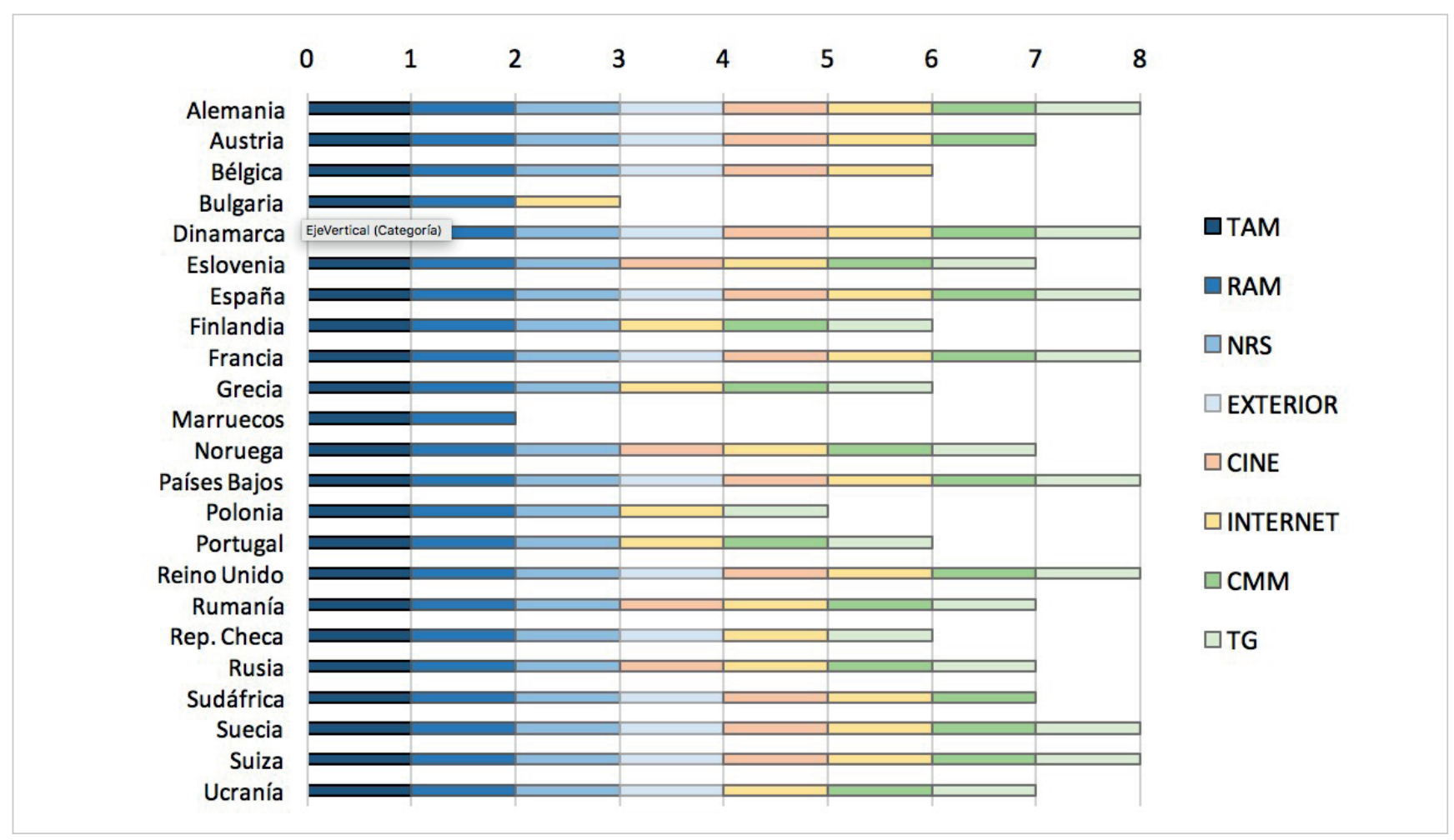

Figura 2. Estudios de audiencias por país

Fuente: elaborado con datos de EMRO (EASI, 2017) 
La mayor parte de los países poseen algún estudio CMM ( $n=18)$ excepto: Bélgica, Bulgaria, Marruecos, Polonia y República Checa. También 18 países disponen de algún estudio que permita la determinación de públicos; de ellos 10 tienen uno específico. Austria, Bélgica, Bulgaria, Marruecos y Sudáfrica no cubren este tipo de medición.

De los 23 países, 8 cubren el $100 \%$ de los estudios recogidos en el inventario: Alemania, Dinamarca, España, Francia, Países Bajos, Reino Unido, Suecia y Suiza (figura 2).

\subsection{Características y dimensiones estudiadas}

Se detectó un grupo de preguntas comunes a cada apartado específico, aunque con singularidades por sección (tabla 2).

Tras un primer bloque de preguntas sobre identificación y descripción de los operadores, se aborda la descripción técnica. En este segundo grupo destacan de cuatro a seis preguntas, según medios. Las cuestiones más básicas giran en torno al tamaño del universo y muestra, presentes en todas las secciones. El método empleado se pregunta en tres casos (RAM, exterior e internet) aunque se puede presuponer en otros dos (TAM, NRS) y deducir en el estudio CMM si se extrapola el caso español a aquellos países que comparten la misma información. Las vías de captación de la muestra están presentes en tres apartados (TAM, RAM y CMM). Casi

Tabla 2. Agrupación y clasificación de las secciones EASI 2017

\begin{tabular}{|c|c|c|c|c|c|c|}
\hline Finalidad & TAM & RAM & NRS & Exterior & Internet & Cross media \\
\hline $\begin{array}{l}\text { Empresa/s que rea- } \\
\text { liza/n el estudio }\end{array}$ & Operador & Operador & Operador & $\begin{array}{l}\text { Operador/ Año } \\
\text { de inicio }\end{array}$ & Operador & Operador \\
\hline \multirow{6}{*}{$\begin{array}{l}\text { Descripción técnica } \\
\text { del estudio }\end{array}$} & $N(H / I)$ & $\mathrm{N}(\mathrm{H} / \mathrm{I})$ & $N(H / I)$ & $N(H / I)$ & $\mathrm{N}(\mathrm{H} / \mathrm{l}, \mathrm{Ho} / \mathrm{lo})$ & $N(H / I)$ \\
\hline & n panel $(\mathrm{H} / \mathrm{I})$ & $\mathrm{n}$ (anual) & n (anual) & $\mathrm{n}(\mathrm{I})$ & $\begin{array}{l}\text { n panel/ muestra } \\
\text { (I) }\end{array}$ & $\mathrm{n}$ (anual) \\
\hline & N.P. & Método (5 tipos) & N.P. & Método & Método (7 tipos) & S.P. \\
\hline & $\begin{array}{l}\text { Captación por } \\
\text { encuesta }\end{array}$ & $\begin{array}{l}\text { Captación (4 técn.+o- } \\
\text { tro)// Reposición }\end{array}$ & S.P. & S.P. & S.P. & $\begin{array}{l}\text { Captación (4 } \\
\text { técn.+otro) }\end{array}$ \\
\hline & $\begin{array}{l}\text { Nombre sist. medi- } \\
\text { ción// Tecnología ( } 5 \\
\text { tipos)//Fusión (site } \\
\text { centric, path data, } \\
\text { surveys) }\end{array}$ & S.P. & $\begin{array}{l}\text { Forma de registro } \\
\text { de datos ( } 4 \\
\text { técn.+otro) }\end{array}$ & Uso del GPS & $\begin{array}{l}\text { Software de } \\
\text { medición }\end{array}$ & $\begin{array}{l}\text { Forma de regis- } \\
\text { tro de datos ( } 5 \\
\text { técn.+otro) }\end{array}$ \\
\hline & N.P. & N.P. & $\begin{array}{l}\text { Filtros// Cálculo } \\
\text { AIR (3 tipos) }\end{array}$ & N.P. & N.P. & N.P. \\
\hline \multirow{2}{*}{$\begin{array}{l}\text { Cobertura de me- } \\
\text { dios y dispositivos }\end{array}$} & N.P. & N.P. & $\begin{array}{l}\text { Tipos de medios } \\
\text { que incluye }\end{array}$ & N.P. & N.P. & $\begin{array}{l}\text { Medios que } \\
\text { incluye }\end{array}$ \\
\hline & S.P. & S.P. & S.P. & S.P. & $\begin{array}{l}\text { Dispositivos me- } \\
\text { didos (4 tipos) }\end{array}$ & $\begin{array}{l}\text { Dispositivos } \\
\text { móviles }\end{array}$ \\
\hline \multirow{2}{*}{$\begin{array}{l}\text { Cobertura de so- } \\
\text { portes y formatos }\end{array}$} & N. de soportes & N. de soportes & N. de soportes & $\begin{array}{l}\text { Clasificación } \\
\text { y mapeo de } \\
\text { soportes }\end{array}$ & $\begin{array}{l}\text { Soportes y } \\
\text { formatos cuyo } \\
\text { tráfico es medido }\end{array}$ & $\begin{array}{l}\text { Si incluye so- } \\
\text { portes }\end{array}$ \\
\hline & $\begin{array}{l}\text { Medición streaming } \\
\text { video por disposi- } \\
\text { tivos }\end{array}$ & $\begin{array}{l}\text { Medición de la escu- } \\
\text { cha online }\end{array}$ & $\begin{array}{l}\text { Medición de } \\
\text { la lectura de la } \\
\text { versión online }\end{array}$ & N.P. & $\begin{array}{l}\text { Medición strea- } \\
\text { ming video por } \\
\text { dispositivos }\end{array}$ & $\begin{array}{l}\text { Medición de la } \\
\text { versión digital de } \\
\text { analógicos }\end{array}$ \\
\hline \multirow{2}{*}{$\begin{array}{l}\text { Otros datos parti- } \\
\text { culares del medio } \\
\text { e intervalos de } \\
\text { medición }\end{array}$} & $\begin{array}{l}\text { Vosdal//Visionado } \\
\text { consolidado por } \\
\text { intervalo }\end{array}$ & S.D. & S.D. & $\begin{array}{l}\text { Tráfico (si/no, } \\
\text { recuent. peato- } \\
\text { nes)//Recons- } \\
\text { truc. rutas }\end{array}$ & S.D. & S.D. \\
\hline & $\begin{array}{l}\text { Medición rating por } \\
\text { intervalo temporal }\end{array}$ & $\begin{array}{l}\text { Intervalo// n. } \\
\text { semanas medidas// } \\
\text { Repetición }\end{array}$ & S.P. & $\begin{array}{l}\text { Frecuencia } \\
\text { actualización } \\
\text { de los datos por } \\
\text { formato }\end{array}$ & S.P. & $\begin{array}{l}\text { Control del } \\
\text { consumo }\left(10^{\prime}, 15^{\prime},\right. \\
\left.30^{\prime}, 60^{\prime}\right)\end{array}$ \\
\hline $\begin{array}{l}\text { Utilidad para la } \\
\text { publicidad }\end{array}$ & S.P. & $\begin{array}{l}\text { Planifica. (software } \\
\text { con datos) }\end{array}$ & $\begin{array}{l}\text { Planifica. (softwa- } \\
\text { re con datos) }\end{array}$ & $\begin{array}{l}\text { Planifica. (medi- } \\
\text { ción frecuencia, } \\
\text { cobertura y VAI) }\end{array}$ & $\begin{array}{l}\text { Planifica. (datos } \\
\text { y frecuencia de } \\
\text { carga) }\end{array}$ & $\begin{array}{l}\text { Planifica. (sof- } \\
\text { tware+ datos } \\
\text { CMM)//Suma } \\
\text { aud. }\end{array}$ \\
\hline $\begin{array}{l}\text { Utilidad para el sec- } \\
\text { tor y publicación }\end{array}$ & $\begin{array}{l}\text { Archivos sin proce- } \\
\text { sar//Publica por tipo } \\
\text { datos// Gráficas }\end{array}$ & $\begin{array}{l}\text { Publicación y fre- } \\
\text { cuencia de la misma }\end{array}$ & $\begin{array}{l}\text { Publicación y } \\
\text { frecuencia de la } \\
\text { misma }\end{array}$ & $\begin{array}{l}\text { 1er año de } \\
\text { publicación y } \\
\text { frecuencia }\end{array}$ & $\begin{array}{l}\text { Frecuencia de la } \\
\text { publicación }\end{array}$ & $\begin{array}{l}\text { Publicación y } \\
\text { frecuencia de la } \\
\text { misma }\end{array}$ \\
\hline \multirow{4}{*}{$\begin{array}{l}\text { Mercado - } \\
\text { Posibilidad de } \\
\text { acceso de datos } \\
\text { para: }\end{array}$} & Medios & Medios & Medios & Medios & Medios & Medios \\
\hline & Agencias & Agencias & Agencias & Agencias & Agencias & Agencias \\
\hline & Anunciantes & Anunciantes & Anunciantes & Anunciantes & Anunciantes & Anunciantes \\
\hline & Otros & Otros & Otros & Otros & Otros & Otros \\
\hline
\end{tabular}

Nota: N: Tamaño universo; n: Tamaño muestra; H/I: Hogares/Individuos; Ho/lo: Hogares con acceso online/Individuos con acceso online; N.P.: No procede; S.P.: Sin pregunta similar, se refiere a que no hay una pregunta semejante a la de las secciones que sí la incluyen, pero podría quedar contenida de forma indirecta en otra; S.D.: Sin otro dato; GPS: Global positioning system; VAl: Visibility adjustment impact. 


\section{$\begin{array}{lllllllllll}0 & 2 & 4 & 6 & 8 & 10 & 12 & 14 & 16 & 18 & 20\end{array}$}

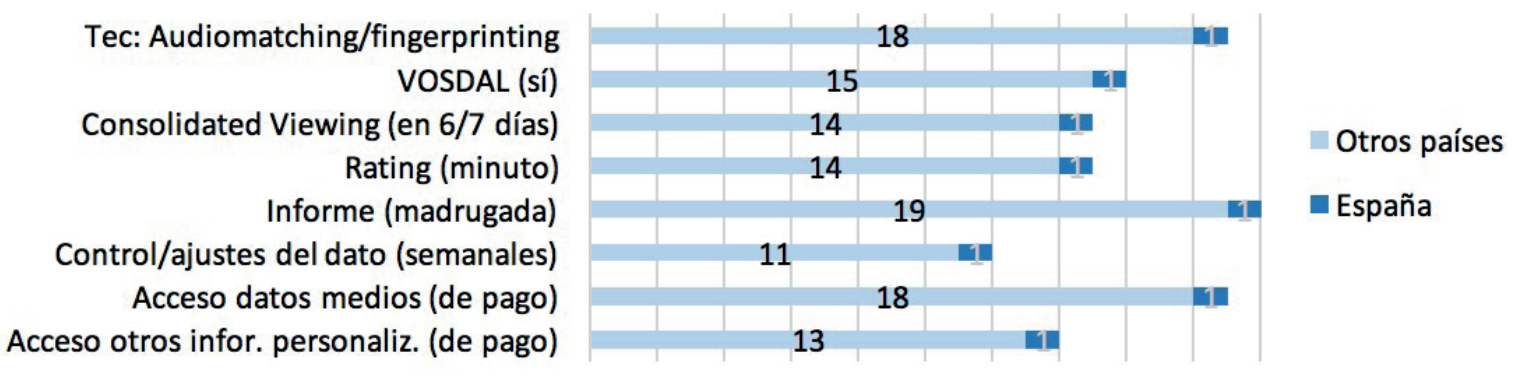

Figura 3. Principales resultados TAM $(n=20)$

Fuente: elaborado con datos de EMRO (EASI, 2017)

todas las secciones contienen alguna pregunta sobre los sistemas de registro y la tecnología utilizada. El medio exterior incluye el uso del GPS (Global positioning system), pues esta tecnología obtiene más información sobre las rutas realizadas. Como técnica propia del medio impreso, también se precisa saber el método para medir el AIR (average issue readership), o lectores de un número medio, y si se incluye una pregunta filtro cuyo fin es mejorar el rendimiento del cuestionario.

\section{La mayoría de países no introducen la retransmision en directo en el estudio de medición de televisión y la mitad lo incluyen en la encuesta de internet}

Un tercer bloque está compuesto por preguntas sobre la cobertura de medios, dispositivos, soportes y algunos formatos de los estudios de un lado, y otras preguntas que se centran en los intervalos de medición y en otros aspectos particulares de cada medio pero relevantes en el contexto actual, de otro.

Finalmente se pueden agrupar en un cuarto bloque las preguntas relacionadas con la utilidad, publicación y comercia- lización de los datos para el sector y para la publicidad y planificación de medios, como muestra la tabla 2.

\subsection{Principales rasgos de la investigación de medios publicitarios clásicos}

En televisión se aprecia que las tecnologías utilizadas por la mayor parte de los países son las conocidas como audiomatching y fingerprinting (figura 3) (95\%). Estas tecnologías permiten reconocer el audio o vídeo que está siendo visionado, por lo que facilitan la comparación con el emitido por el canal (en directo o en diferido) y en consecuencia, su identificación. En relación con la medición de la audiencia en diferido se observa que la mayor parte de los países la miden dentro del día de emisión del vídeo, que es la definición de Vosdal (80\%). También estiman los visionados de los programas retransmitidos en directo o en diferido al cabo de una semana (75\%). En cuanto al rating o audiencia expresada en porcentajes, el minuto es la media convenida por muchos países para su cálculo (75\%). Finalmente en este medio la mayor parte de los territorios ponen los resultados a disposición de las cadenas previo pago. Todos generan informes diarios y algunos aplican además controles o ajustes semanales.

En radio el $76 \%$ aplica el método recall, que utiliza la encuesta y se basa en el recuerdo (figura 4). La modalidad telefóni-

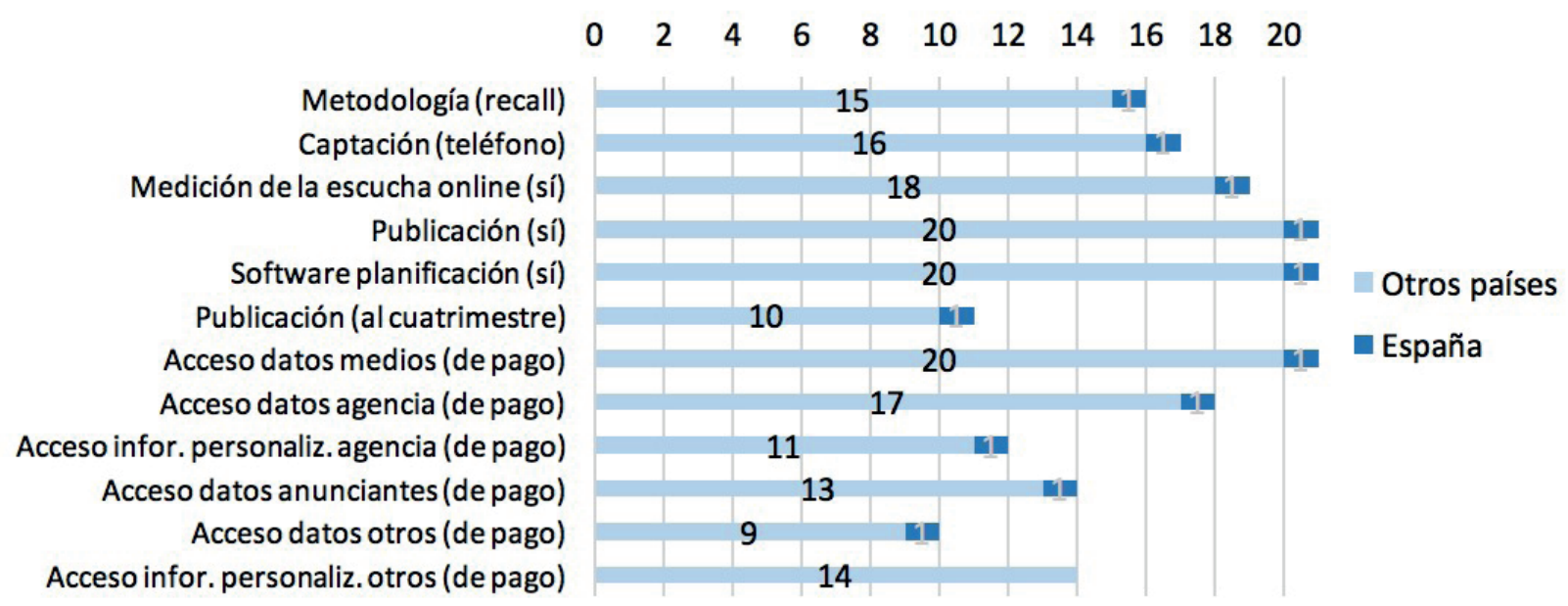

Figura 4. Principales resultados RAM $(n=21)$

Fuente: elaborado con datos de EMRO (EASI, 2017) 


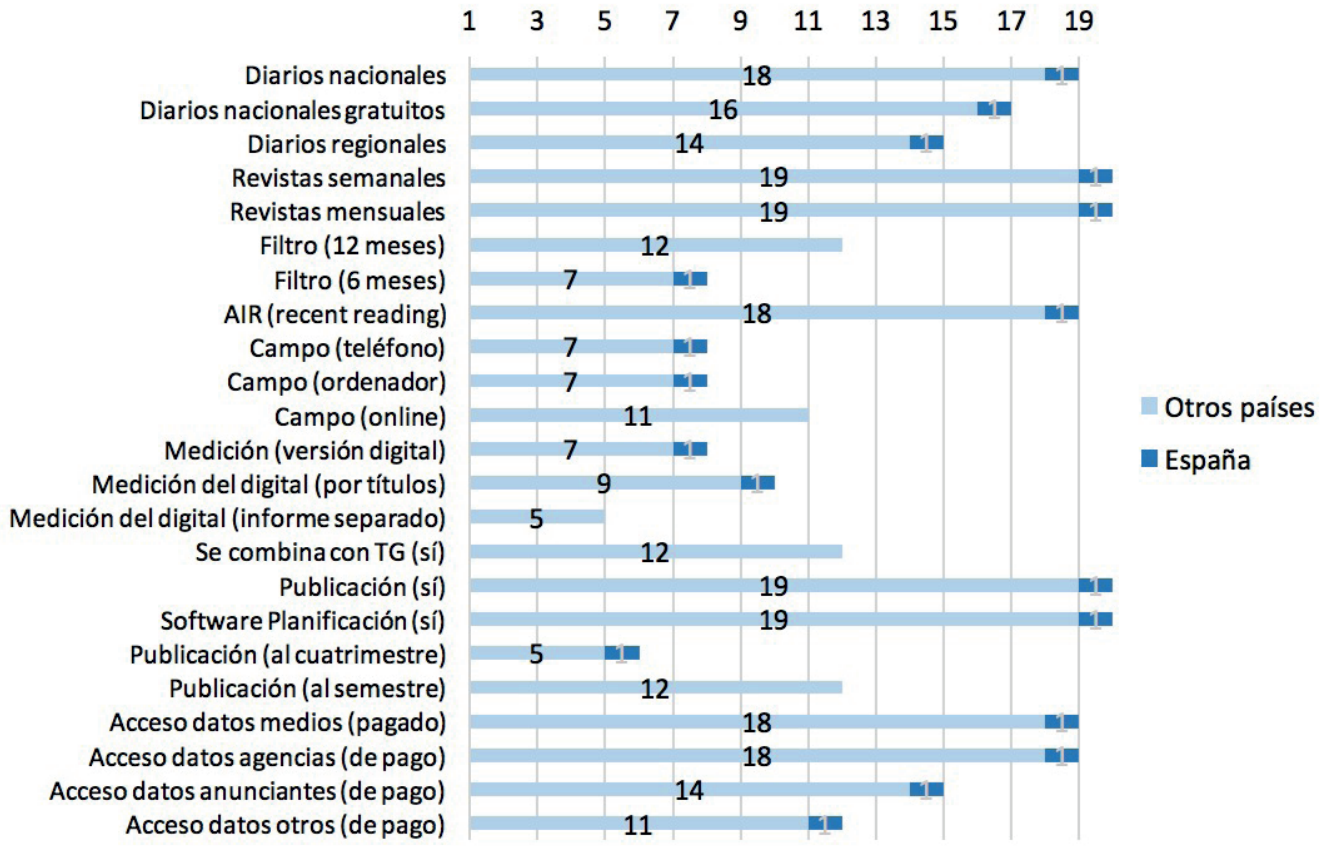

Figura 5. Principales resultados NRS $(n=20)$

Fuente: elaborado con datos de EMRO (EASI, 2017)

ca es la más extendida, también en el caso de España al tratarse de una de las ampliaciones monográficas del Estudio General de Medios (EGM). El 90\% de los países afirma medir la escucha online. La preparación de los datos para ser utilizados en la publicidad está presente en la totalidad de los países. La orientación hacia el sector y el mercado también es evidente si se observan la publicación y el acceso pagado a los datos. Menos países disponen de publicaciones cuatrimestrales o facilitan otro tipo de informes.

\section{La vocación por conocer a la audiencia aparece en el origen de cualquier medio}

En el medio impreso la mayoría cuenta con estudios que incluyen diarios y revistas y diferentes tipos de soportes (figura 5). Todos estiman el AIR con el método recent reading (usa la encuesta y se basa en el recuerdo). El $60 \%$ de los países mejoran este método con una pregunta previa al AIR que consigue filtrar los títulos de las revistas que no recuerdan haber sido leídos en los últimos 12 meses. En España y en otros siete países, esta pregunta se centra en los últimos 6 meses para las revistas. También hay diferencias en el trabajo de campo español. El $60 \%$ de los países utiliza la modalidad online para conocer las audiencias del medio impreso, pero en España se combina la telefónica con la entrevista cara a cara asistida con ordenador (CAPI - computer assisted personal interviewing) pues obedece al diseño del EGM.

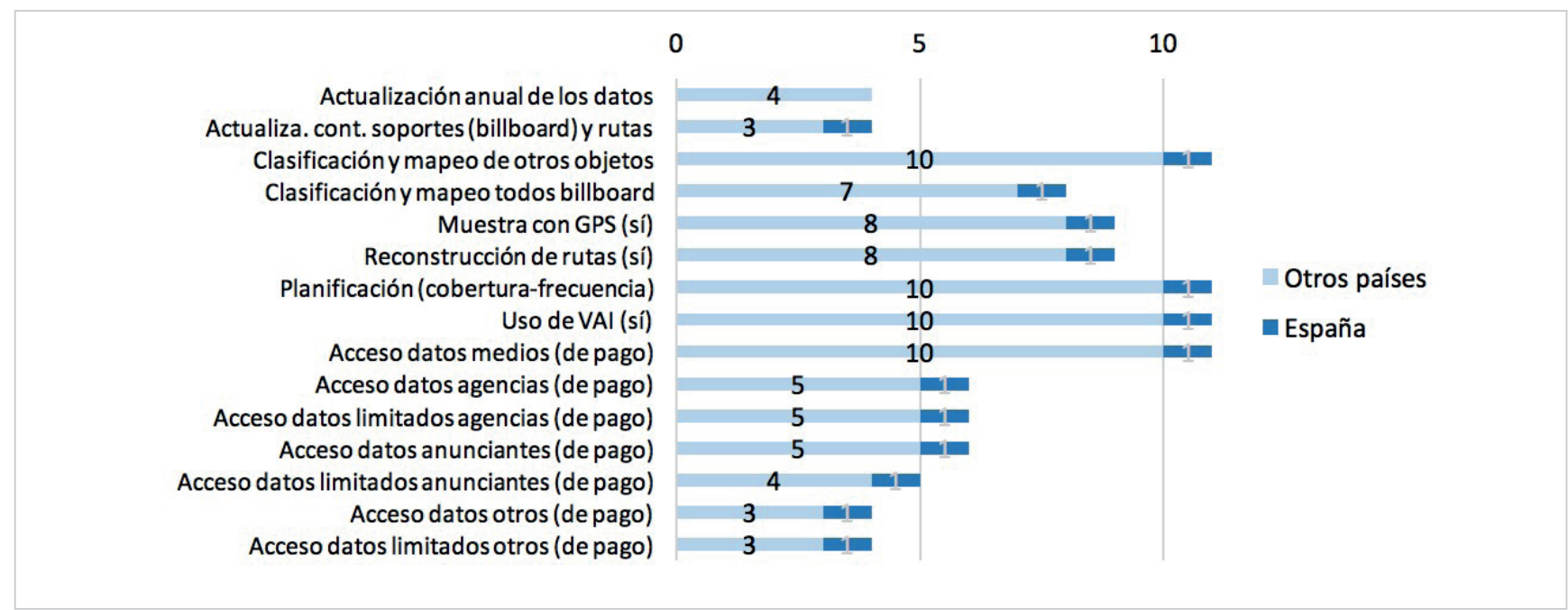

Figura 6. Principales resultados Exterior $(n=11)$

Fuente: elaborado con datos de $E M R O$ (EASI, 2017) 


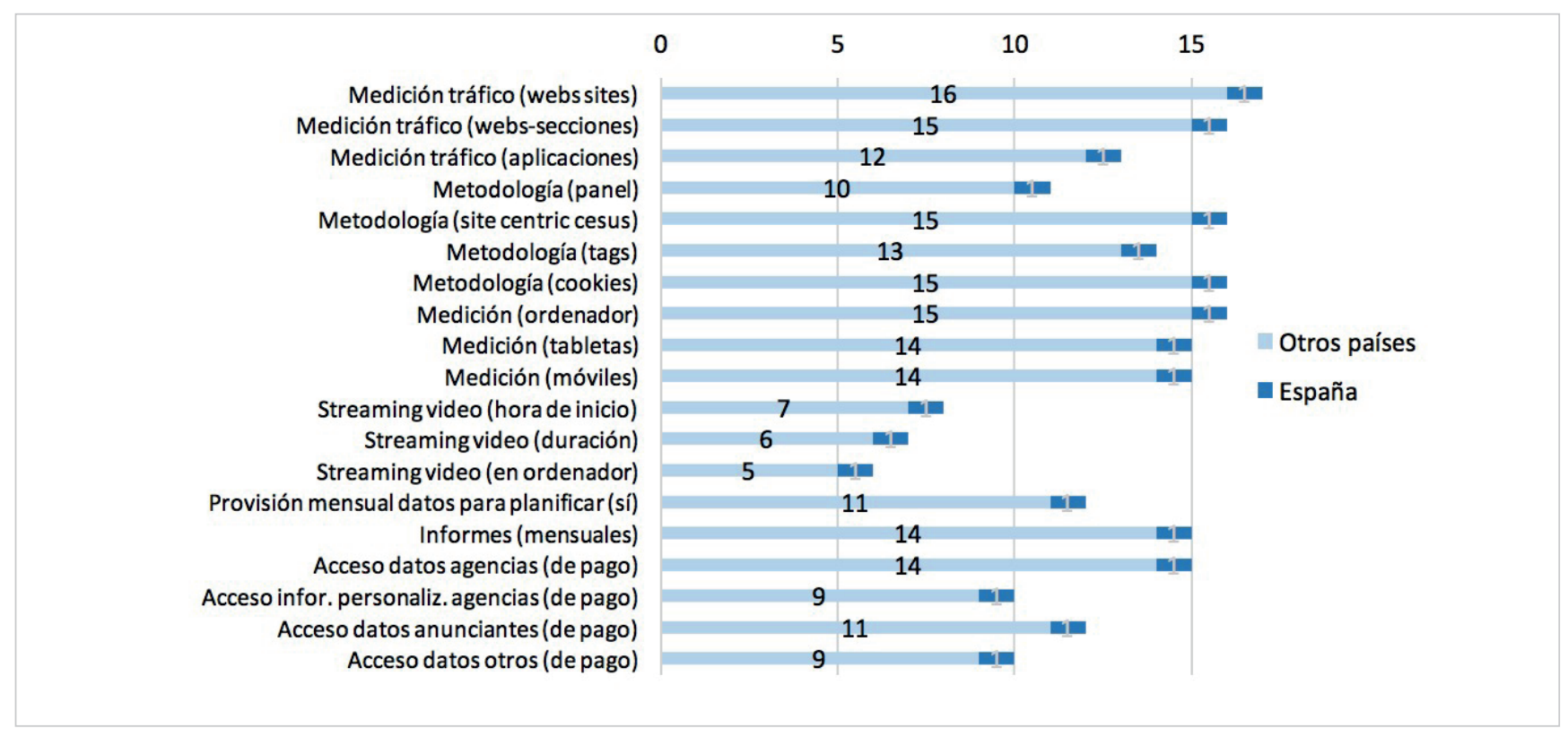

Figura 7. Principales resultados Internet $(n=17)$

Fuente: elaborado con datos de EMRO (EASI, 2017)

España dispone además de un estudio TG independiente: AIMC marcas. También forma parte de los países que miden la versión online del medio impreso, aunque no emite un informe separado. La preparación de los resultados para la publicidad y la orientación hacia el sector y el mercado sigue la misma pauta que con la radio.

Menos de la mitad de países afirman disponer de un estudio monográfico del medio exterior; España es uno de ellos. La actualización de la información en estos estudios no siempre es anual, puede depender del área geográfica y tener una frecuencia en su conjunto superior o inferior al año (figura 6). La actualización de los soportes y de las rutas tampoco es habitual, pero en este caso el estudio español se sitúa entre los que revisan el inventario antes del año. Una parte relevante de estos estudios clasifican y ubican geográficamente paneles publicitarios, además de otro tipo de objetos. El $82 \%$ reconstruye las rutas atendiendo al punto de partida y de llegada. El mismo porcentaje hace uso del GPS para conocer con mayor exactitud los trayectos realizados por los individuos. Todos tienen en cuenta el VAI (visibility adjustment impact) que responde al grado de visibilidad del anuncio publicitario. Igualmente todos los países que contestan esta sección ofrecen estimaciones de cobertura y de frecuencia útiles para la publicidad. También proporcionan información a las empresas de medios previo pago. Algunos se orientan hacia las agencias y anunciantes y realizan otro tipo de informes.

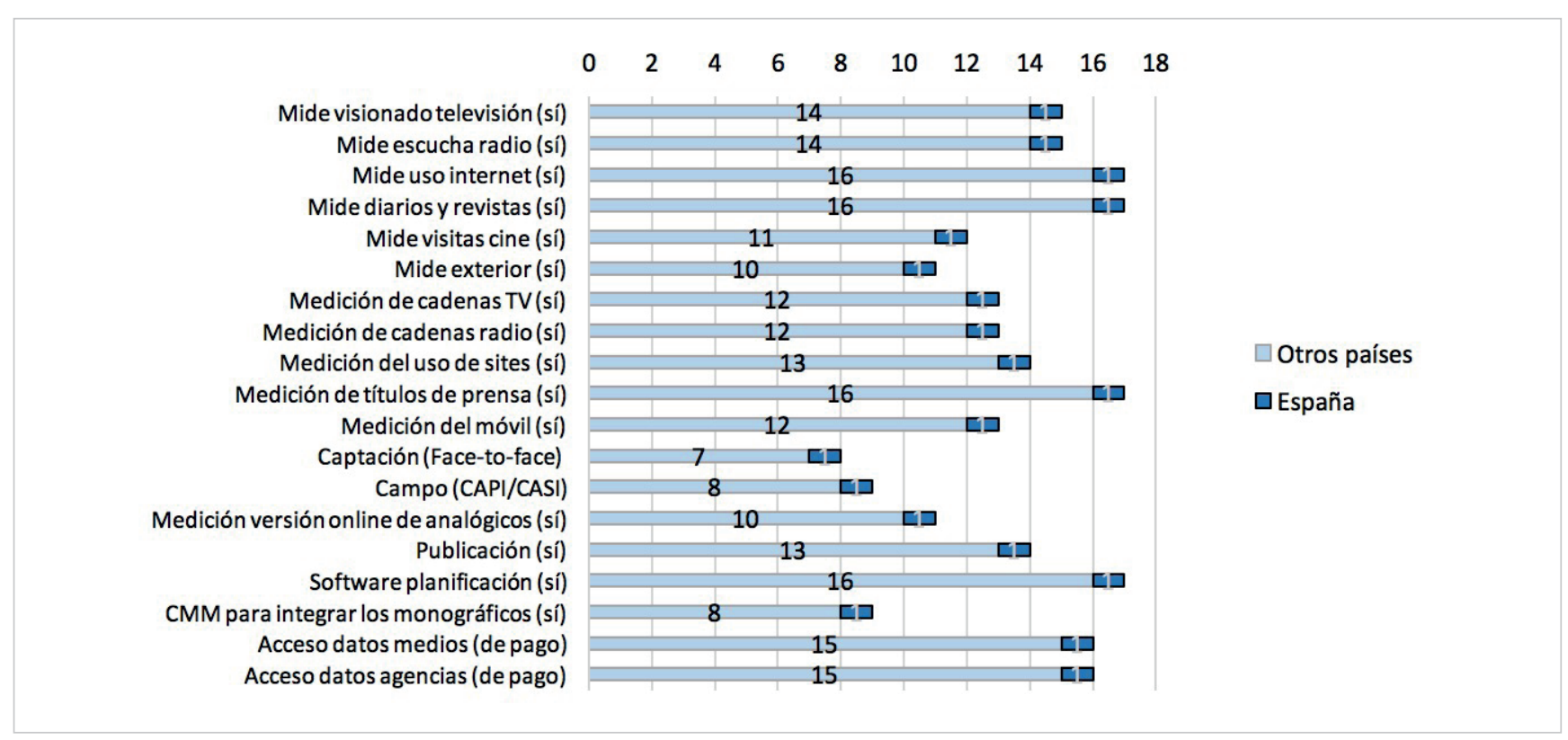

Figura 8. Principales resultados $\mathrm{CMM}(\mathrm{n}=17)$

Fuente: elaborado con datos de EMRO (EASI, 2017) 


\subsection{Principales rasgos del estudio online y cross media}

Los países que disponen de un estudio de audiencias online ofrecen cifras de tráfico de los sitios web y de sus secciones (figura 7). Un gran número de ellos también lo hacen de aplicaciones (apps). En 11 casos utilizan el panel, la fórmula híbrida. La gran mayoría tiene un sistema censal (electrónico) de registro de datos, a través de cookies y tags. En cuanto a dispositivos, miden ordenadores, tablets y teléfonos. En menor medida se conoce el consumo del vídeo, aunque España incorpora esta pregunta. Los resultados también se orientan hacia la planificación en el $71 \%$ de los casos. Los informes realizados tienen una frecuencia mensual y previo pago los datos son puestos a disposición de las agencias e incluso de los anunciantes.

\section{El modelo debe estimar el espacio pro- pio de las sociedades digitales: la expo- sición de cualquier contenido, en cual- quier momento, lugar y dispositivo}

Los estudios CMM más extendidos son aquellos que miden el uso de internet, diarios y revistas. También se apuesta por medios audiovisuales como televisión y radio y en menor medida, el cine y exterior (figura 8). Aunque con diferente presencia, proporcionan información sobre los soportes de los medios mencionados. La captación de la muestra es cara a cara y el campo se realiza aplicando la modalidad CAPI o CASI (self-interviewing). La orientación hacia la publicidad se hace patente en todos los países. Asimismo se apuesta por la publicación y el acceso para medios y agencias previo pago. En el 53\% de los casos los datos CMM son utilizados para fusionar los estudios monográficos y disponer de una audiencia única.

\section{Conclusiones}

En términos generales es satisfactoria la cobertura de estudios por medio y país, aunque se detectan algunos países cuya investigación de audiencias consiste únicamente en dos o tres estudios, como Marruecos y Bulgaria. Los medios más observados son televisión, radio, medio impreso e incluso internet. Prácticamente todos los países tienen un estudio CMM. La medición del medio exterior presenta en cambio menor cobertura internacional, quizá debido a la dificultad que entraña (Lamas, 2002).

De los resultados del análisis cualitativo y cuantitativo de las secciones se desprende que la investigación de audiencias responde a las necesidades de la publicidad en los procesos offline. En estos casos algunas mediciones que son interesantes para los medios de comunicación como empresas son claves para la toma de decisiones sobre la publicidad. La importancia del acceso a los datos, tanto por las empresas informativas como por la publicidad, se refleja en el inventario. Todas las secciones específicas incluyen una serie de preguntas orientadas a la planificación de los medios, a la publicación y tipo de acceso a los resultados del estudio. Dichas cuestiones sobresalen como rasgos clave en las respuestas de la gran mayoría de los países.
También se observan avances hacia la medición de los retos presentados por la transformación digital. Se pueden destacar al menos cuatro:

- alta presencia de los estudios del medio internet;

- introducción de preguntas sobre versiones digitales;

- enfoque cross media;

- presencia de diversos dispositivos y formatos digitales.

En primer lugar, la evidencia más sobresaliente es la existencia de un estudio centrado en internet en 17 de los 23 países. Predomina la medición electrónica (basada en el censo), aunque existe un gran número que utiliza también la centrada en el usuario. Esta parte pregunta por la medición no sólo de los sitios webs sino también de sus secciones; consideración central para conocer el nivel de precisión del estudio en este medio. Igualmente incluye la medición del tráfico procedente de las apps y de diferentes pantallas. Estas preguntas capturan los principales aspectos del debate sobre la medición de internet pero además son contestadas afirmativamente por un gran número de países. Es más, si se observan los resultados de las preguntas dirigidas a averiguar la utilidad y el acceso a la información de este estudio en comparación con las contestadas en otros medios, se aprecia cómo desaparece la orientación hacia las empresas informativas y aparece el anunciante como destinatario directo. Este resultado podría estar reflejando la peculiaridad que introduce este medio sobre el papel de los soportes para la publicidad, pues cada vez es menor la necesidad de disponer de datos sobre los mismos para poder planificar la publicidad online. Internet facilita métricas generadas directamente por las audiencias y hace posible que el anuncio aparezca allí donde se encuentre el público conectado.

\section{Casi todos los países tienen un estudio} $\mathrm{CMM}$, la mayoría miden el uso de internet, los diarios, revistas y medios audiovisuales. Cine y exterior aparecen en último lugar

En segundo lugar, las secciones centradas en radio, medio impreso y estudios CMM incluyen preguntas sobre la medición de las versiones digitales online. Parece evidente que se deba al alto grado de participación de los tradicionales en internet, pero también facilita conocer el consumo entre medios distintos de la población. En planificación, el dato de consumo multimedia es central para poder hacer mejores estimaciones de cobertura y frecuencia.

En tercer lugar es necesaria la sección que pregunta sobre las fuentes CMM de los países y en mayor medida si incluye internet, como es el caso. En especial se incorpora el uso de los datos cross media para la fusión de los diferentes estudios monográficos, cuestión clave. Además, el estudio CMM pregunta por la medición del móvil como dispositivo estrella de navegación. El teléfono vuelve a ocupar un lugar central en la sección general del inventario y aparece junto a otros dispositivos en otras secciones, como en televisión, al preguntar por la exposición a un formato específico, el vídeo. 
De acuerdo con los recientes estudios sobre visionado de este formato, la presencia del vídeo estaría justificada (ZenithMedia, 2017).

En consecuencia y en cuarto lugar se señala la introducción de los ordenadores, tablets y móviles en varias secciones: general, televisión, internet y multimedia, pues facilitan la observación multipantalla. Igualmente se destaca la presencia de formatos específicos audiovisuales vinculados a los servicios digitales, como el vídeo online y el consumo en diferido.

A juzgar por la información que recoge el inventario, los datos también presentan limitaciones para poder conocer el comportamiento de todas las audiencias y facilitar la planificación de medios publicitarios. En concreto no quedan suficientemente reflejadas las audiencias sociales o la exposición desde otras pantallas, como las que forman el digital signage (medio exterior digital y otra publicidad interior), o tipos de soporte en internet. Se siguen apreciando intervalos de tiempo elevados para la publicidad, como las estimaciones sobre el minuto en televisión o la generación de informes mensuales en internet. A todo lo anterior hay que añadir que se desconocen los detalles de las mediciones incluidas en la fuente que pudieran desvelar otros vacíos de información para comprender el escenario digital. También deben considerarse las desventajas de las encuestas en la producción de datos digitales y la necesidad de desarrollar tecnologías para poder superar las de los sistemas basados en el censo, que siguen siendo los principales retos de la medición de audiencias.

La orientación hacia la publicidad y la planificación de medios es común en todas las mediciones estudiadas

España está entre los ocho países mejor situados: la investigación de audiencias contiene todos los estudios recogidos en el inventario de $E M R O$ sin excepción. Al profundizar en las secciones específicas, su situación tampoco empeora comparativamente $y$, aunque se detectan algunas singularidades que podrían ser abordadas en futuras propuestas, algunos de los desafíos son mayores en otros países si se observan los vacíos de información.

A modo de cierre, se aprecian avances para ajustar el modelo tradicional de medición de audiencias al escenario digital online, pero sigue presentando limitaciones desde una perspectiva internacional. Se esperan cambios más profundos precipitados por el desarrollo tecnológico de la era de los datos.

\section{Notas:}

1. AIMC es un referente de la investigación de medios en España, tanto por su trayectoria histórica como por la labor que desempeña en el impulso de la medición de medios en la actualidad (por ejemplo, el 22 de diciembre de 2017 convoca el nuevo concurso para la medición online en este país). Pertenece a la organización transnacional I-JIC (The International Association of Joint Industry Committees for Media Research) y a Esomar, entidades con las que completaría su presencia y proyección internacional. http://www.aimc.es/informacion-y-formacion/relacionesinternacionales

Tras una previa exploración de las páginas de las tres asociaciones, $E A S I$ de $E M R O$ es la fuente que facilita la observación internacional pretendida por este estudio.

2. EMRO ofrece la información de EASI en hoja de cálculo. Se puede acceder al mismo en este enlace: https://www.emro.org/easi.html

\section{Referencias}

Campos-Freire, Francisco (2015). "Adaptación de los medios tradicionales a la innovación de los metamedios". El profesional de la información, v. 24, n. 4, pp. 441-450. https://doi.org/10.3145/epi.2015.jul.11

González-Lobo, María-Ángeles; Carrero-López, Enrique (2008). Manual de planificación de medios publicitarios. (5a ed. rev.). Madrid: Esic. ISBN: 9788473565233

Gonzalez-del-Valle, Almudena (2003). “Medición de audiencias de televisión en España: una perspectiva histórica". En: Igartua-Perosanz, Juan-José; Badillo-Matos, Ángel (Eds.). Audiencias y medios de comunicación, pp. 43-55. Salamanca: Universidad de Salamanca. ISBN 9788478007257

Hill, Shawndra (2014). "TV audience measurement with big data". Big data, v. 2, n. 2, pp. 76-86. https://doi.org/10.1089/big.2014.0012

Huertas, Amparo (2002). La audiencia investigada. Barcelona: Gedisa. ISBN 9788474329575

Lamas, Carlos (2002). "El uso de la tecnología en la medición de audiencias". AIMC. http://www.aimc.es/a1mc-c0nt3nt/uploads/2010/10/EI_ uso_de_la_tecnologia.pdf

Lamas, Carlos (2004). "La medición de la audiencia de los medios: una visión actualizada". Revista el publicista, n. 101. http://www.aimc.es/a1mc-c0nt3nt/uploads/2010/10/ medicion_audiencia_el_publicista.pdf

Lamas, Carlos (2010). "Los medios interactivos y su publicidad. La medición de audiencias". Telos. Cuadernos de comunicación e innovación, n. 82, pp. 95-101. https://goo.gl/b98iwA

Livingstone, Sonia (2004). "The challenge of changing audiences: or, what is the audience researcher to do in the age of the internet?". European journal of communication, v. 19, n. 1. pp. 75-86.

http://eprints.Ise.ac.uk/412

Madinaveitia, Eduardo (2013). “¿Tiene futuro la televisión conectada?". Ipmark, 12 julio.

http://ipmark.com/tiene-futuro-la-televisin-conectada/

Madinaveitia, Eduardo; Merchante, Mapi (2015). “Medición de audiencias: desafío y complejidad en el entorno digital". Harvard Deusto marketing y ventas, n. 131, pp. 26-33.

Masip, Pere (2016). "Investigar el periodismo desde la perspectiva de las audiencias". El profesional de la información, v. 25 , n. 3, pp. 323-330. 
https://doi.org/10.3145/epi.2016.may.01

Nelson, Jacob L.; Webster, James G. (2016). "Audience currencies of the age of big data". International journal on media management, v. 18, n. 1, pp. 9-24.

https://doi.org/10.1080/14241277.2016.1166430

O’Hara, Chris (2016). “Data triangulation: How second-party data will eat the digital world". AdExchanger, January 25. https://goo.gl/b7KbEr

Papí-Gálvez, Natalia (2014). "Los medios online y la ¿crisis? de la planificación de medios publicitarios". adComunica. Revista científica de estrategias, tendencias e innovación en comunicación, n. 7, pp. 29-48.

http://www.adcomunicarevista.com/ojs/index.php/ adcomunica/article/view/166

Papí-Gálvez, Natalia (2015a). “Las audiencias televisivas y las audiencias online en publicidad: ¿diferencias y semejanzas?". En: Quintas-Froufe, Natalia; González-Neira, Ana (coord.), La participación de la audiencia en la televisión: de la audiencia activa a la social, pp. 148-166. Madrid: AIMC. http://rua.ua.es/dspace/handle/10045/52397

Papí-Gálvez, Natalia (2015b). "Los nuevos medios y las empresas innovadoras. El caso de las agencias de medios". El profesional de la información, v. 24, n. 3, pp. 301-309. https://doi.org/10.3145/epi.2015.may.10
Papí-Gálvez, Natalia (2017). Investigación y planificación de medios publicitarios. Madrid: Editorial Síntesis. ISBN 97884 91710844

Perlado-Lamo-de-Espinosa, Marta (2006). Planificación de medios de comunicación de masas. Madrid: McGrawHill. ISBN 9788448142100

Sánchez, Poli (2017). "La medición de audiencias en la encrucijada". IPMark, 12 abril.

http://ipmark.com/medios-de-comunicacion-medicionaudiencia

Santiago, Fernando (2017). "Fragmentación de la audiencia. Retos de la medición". En Jornada OCC Incom UAB, junio. http://www.aimc.es/a1mc-c0nt3nt/uploads/2017/09/170609_ fsc_jornada_occ_barcelona-2.pdf

Solana, Daniel (2010). "Internet. El medio rey". Telos. Cuadernos de comunicación e innovación, n. 82, https://goo.gl/3jioH7

Wimmer, Roger D.; Dominick, Joseph R. (2001). La investigación científica de los medios de comunicación. Una introducción a sus métodos. Barcelona: Bosch Casa Editorial. ISBN 9788476763599

ZenithMedia (2017). Zenith 2017 Online Video Forecast. https://goo.gl/gYK4iY

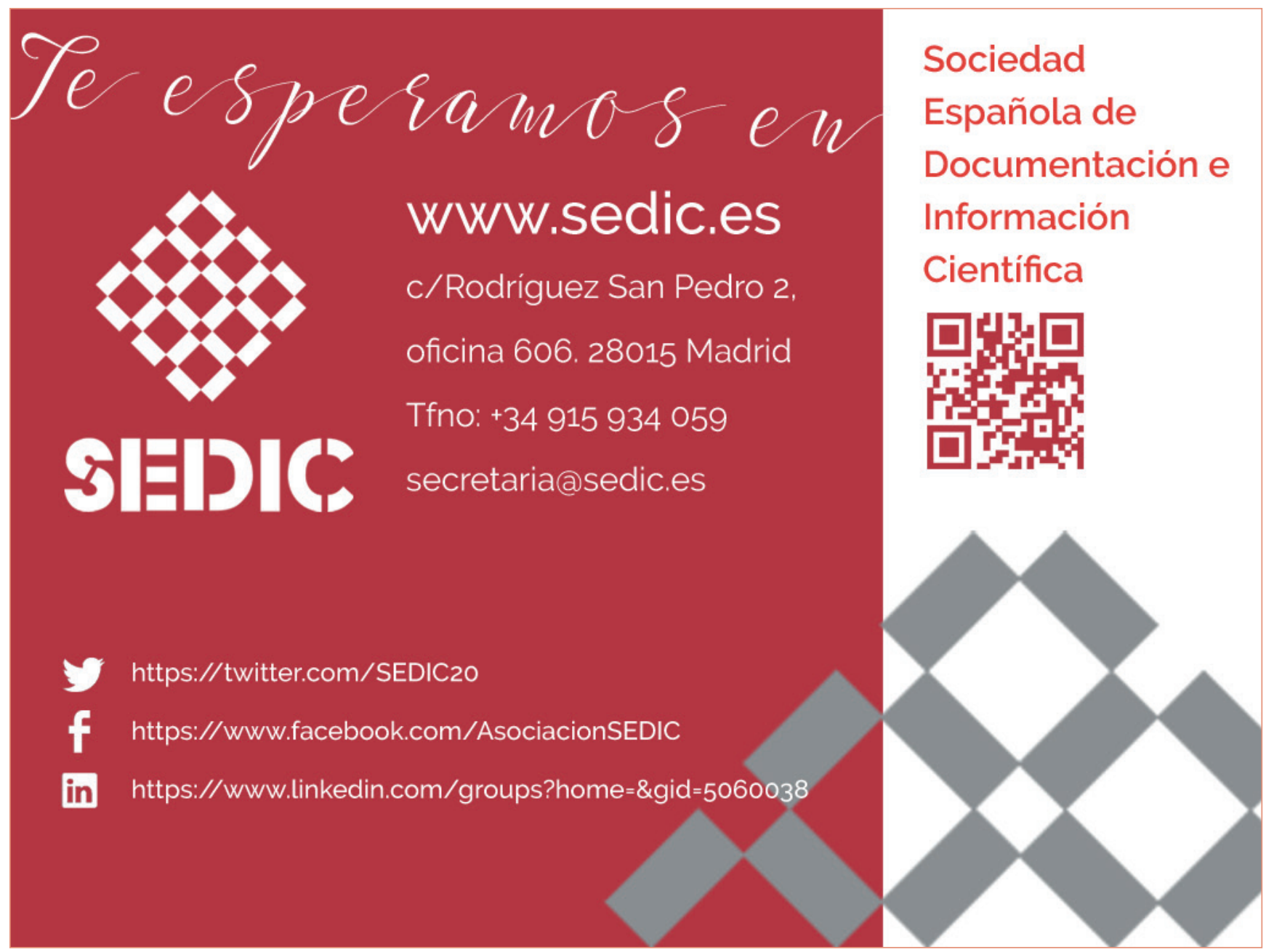




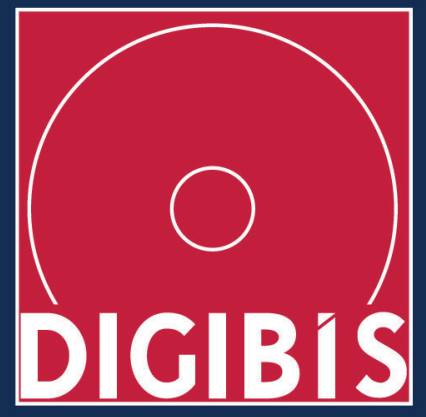

Nuestra vocación: contribuir a que el patrimonio cultural español sea preservado digitalmente y se difunda de la manera más amplia posible

Con metadatos ajustados a la normativa internacional

Aplicaciones Linked Open Data (LOD) con Reconciliación Semántica

Aplicaciones que pueden incorporar Recolector y Repositorio OAI-PMH

Aplicaciones pensadas y desarrolladas para hacer más eficiente y ágil el trabajo de los administradores y más comprensible para el usuario el acceso a los recursos catalogados. 